\title{
On the Origin of Iron $\mathrm{K} \alpha$ and $\mathrm{HI}$ Balmer Lines in AGN
}

\author{
P. Marziani and M. Calvani \\ Osservatorio Astronomico di Padova, Italia \\ J. W. Sulentic, P. Romano, T. Zwitter and D. Dultzin-Hacyan \\ University of Alabama, Tuscaloosa, USA
}

\begin{abstract}
The idea that a significant fraction of line emission in AGN comes directly from an accretion disk is attractive for several reasons not least the obvious one of establishing the black hole paradigm. We performed a few consistency tests for the hypothesis that both FE K $\alpha$ and $\mathrm{H} \alpha$ are emitted by an accretion disk.

The main results of this preliminary investigation are as follows. $\mathrm{FE}$ $\mathrm{K} \alpha$ is systematically much broader than $\mathrm{H} \alpha$ at FWHM, but the widths of the two lines are not correlated. For each of the 9 objects with FE K $\alpha$ profile most consistent with disk emission, we computed the possible disk contribution on the basis of a weak field model. Although the $\mathrm{H} \alpha$ profiles are not consistent with disk model emission, rotational motion could still be the main broadening mechanism. We then compared disk inclinations deduced from $\mathrm{FE} \mathrm{K} \alpha$ model profile fitting to the ones we derived from model fits of $\mathrm{H} \alpha$. The inclination values are consistent. Finally, we found that a simple illumination model can produce both disk $\mathrm{FE} \mathrm{K} \alpha$ and $\mathrm{H} \alpha$ emission, if a small fraction of continuum is scattered toward the disk to yield ionization parameter $\xi>10^{-2} \mathrm{ergs} \mathrm{s}^{-1} \mathrm{~cm}$.

We conclude that the bulk FE K $\alpha$ cannot be associated to the Broad Line Region. Albeit several radio-quiet Seyfert 1 objects show $\mathrm{FE} K \alpha$ profiles consistent with emission from the inner part of an illuminated accretion disk, the general situation remains unclear, as fits to line profiles require an unsatisfactory large range in model parameters like emissivity and inner and outer radii.
\end{abstract}

